\title{
Article
}

\section{Mechanical effects of medial and lateral wedged orthoses during running}

Sinclair, Jonathan Kenneth

Available at http://clok.uclan.ac.uk/22512/

Sinclair, Jonathan Kenneth ORCID: 0000-0002-2231-3732 (2018) Mechanical effects of medial and lateral wedged orthoses during running. Physical Therapy in Sport, 32 . pp. 48-53. ISSN 1466-853X

It is advisable to refer to the publisher's version if you intend to cite from the work. http://dx.doi.org/10.1016/j.ptsp.2018.04.020

For more information about UCLan's research in this area go to http://www.uclan.ac.uk/researchgroups/ and search for < name of research Group>.

For information about Research generally at UCLan please go to http://www.uclan.ac.uk/research/

All outputs in CLoK are protected by Intellectual Property Rights law, including Copyright law. Copyright, IPR and Moral Rights for the works on this site are retained by the individual authors and/or other copyright owners. Terms and conditions for use of this material are defined in the policies page.

\section{CLoK}

Central Lancashire online Knowledge www.clok.uclan.ac.uk

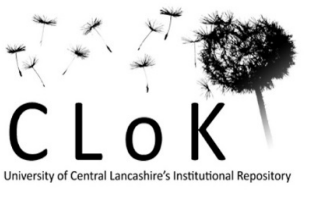


5 Keywords: Orthoses, Biomechanics, Running, Knee, Joint moments.

\section{Abstract}

7 OBJECTIVE: The aim of the current investigation was to examine the effects of orthoses

\section{Jonathan Sinclair ${ }^{1}$}

1. Centre for Applied Sport and Exercise Sciences, School of Sport and Wellbeing, Faculty of Health \& Wellbeing, University of Central Lancashire, Lancashire, UK.

with $5^{\circ}$ medial and lateral wedges on knee joint kinetics during the stance phase of running.

DESIGN: Repeated measures

\section{SETTING: Laboratory}

\section{PARTICIPANTS: Twelve recreational runners}

2 OUTCOME MEASUREMENTS: Twelve male participants ran over a force platform at 4.0 $\mathrm{m} / \mathrm{s}$ in three different conditions (medial orthotic, lateral orthotic and no-orthotic). Lower limb kinematics were collected using an 8-camera motion capture system allowing knee kinetics to be quantified using a musculoskeletal modelling approach. Differences in knee joint kinetics between orthotic conditions were examined using one-way repeated measures ANOVA.

RESULTS: The results showed that peak patellofemoral force was significantly increased in the medial $(31.81 \mathrm{~N} / \mathrm{kg})$ and lateral $(31.29 \mathrm{~N} / \mathrm{kg})$ wedged orthoses, in comparison to the noorthotic $(29.61 \mathrm{~N} / \mathrm{kg})$ condition. In addition, the peak knee adduction moment was significantly increased in the medial $(1.10 \mathrm{Nm} / \mathrm{kg})$ orthoses, in comparison to the lateral $(0.87$ $\mathrm{Nm} / \mathrm{kg}$ ) condition. 
23 CONCLUSIONS: The results from this study indicate that lateral orthoses may be effective

24 in attenuating runners risk from medial tibiofemoral compartment OA, but that wedged

25 orthoses may enhance their risk from patellofemoral pain.

\section{Introduction}

Although distance running is associated with a plethora of physiological benefits (Lee et al., 2014), it is also linked with a very high rate of overuse injuries (Taunton et al., 2002), with an occurrence rate of up to $70 \%$ per year of training (Van Gent et al., 2007). The knee joint is the musculoskeletal site that is most likely to experience an overuse injury (van Gent et al., 2007). Specifically, patellofemoral pain and pain secondary to knee osteoarthritis are common complaints reported by runners (Taunton et al., 2002).

Patellofemoral pain syndrome is regarded as the most common overuse injury in runners

(Taunton et al., 2002). Pain symptoms present clinically as isolated pain at the anterior aspect

of the patella (Ho et al., 2012). As knee flexion proceeds from full extension, the pull of the quadriceps and patellar tendon becomes increasingly oblique, compressing the patella against the femur and generating the patellofemoral joint reaction force (Trepczynski et al., 2012). The dynamics of the knee in the sagittal plane may have a prominent effect on the patellofemoral joint, and a model to estimate the patellofemoral joint reaction force has previously been developed (Ward \& Powers, 2004). Elevated patellofemoral joint stress, which is a reflection of the patellofemoral joint reaction force divided by the patellofemoral contact area, is the most commonly accepted aetiological factor in the development of 

adduction (Barton et al., 2011). These mechanisms are thought enhance patellofemoral stress, owing to a reduced joint contact area (Tiberio, 1987). Patellofemoral pain symptoms can

49

cause training restrictions (Waryasz \& McDermott, 2008), and pain symptoms associated with patellofemoral disorders can persist for many years (Collins et al., 2013). Importantly, 45-64\% of individuals with patellofemoral osteoarthritis (OA) report patellofemoral pain symptoms during adolescence or early adulthood (Crossley, 2014).

Degenerative tibiofemoral pathologies are also common in runners; accounting for as many as many as $16.8 \%$ of all chronic knee injuries (Taunton et al., 2002). The causes of tibiofemoral chronic pathologies relate to the magnitude of compressive loading at the joint (Morgenroth et al., 2014), which is considered to be the mechanical parameter most strongly associated with the onset and progression of knee OA. The medial aspect of the tibiofemoral joint is known to be significantly more prone to osteoarthritic degeneration than the lateral compartment (Wise et al., 2012). In vivo analyses have shown that compressive loading experienced by the medial aspect of the tibiofemoral joint is correlated positively with the magnitude of the knee adduction moment (KAM) (Zhao et al., 2007; Kutzner et al., 2013). Therefore, the KAM is frequently utilized as a pseudo measure of medial tibiofmeoral contact loading (Birmingham et al., 2007), and the peak KAM as well as the slope of the KAM have been cited as important predictors of radiographic knee OA (Miyazaki et al., 2002; Morgenroth et al., 2014).

Given their prevalence and debilitating nature, numerous strategies have been investigated in clinical research in an attempt to attenuate the risk of knee pathologies in runners. Foot 
70 orthoses are one of the most popular conservative options for the prevention/ treatment of

71 knee pathologies in runners (Heiderscheit et al., 2001). For patellofemoral pain symptoms,

72 foot orthoses have importantly, been shown to be successful in improving pain symptoms and

73 function (Collins et al., 2008; Barton et al., 2011).

In addition to traditional foot orthoses, wedged orthoses that are built up along either the medial or lateral edges have become common in recent years (Aminian et al., 2014). Medially orientated foot orthoses are often utilized to reduce lower extremity biomechanics linked to increases in patellofemoral stress by attenuating rearfoot eversion/ tibial internal rotation during the stance phase (Boldt et al., 2013). However, using a sagittal plane model to estimate the patellofemoral loading, Almonroeder et al., (2015), showed that prefabricated

foot orthoses with $5^{\circ}$ of medial rearfoot wedging significantly increased peak patellofemoral

stress compared to running without orthoses. Similarly, laterally wedged orthoses have also been advocated as a mechanism that may reduce the magnitude of the KAM and thus the loads experienced by the medial compartment of the tibiofemoral joint (Yamaguchi et al., 2015). Lewinson et al., (2013) who investigated the effects of 3, 6, and $9 \mathrm{~mm}$ medial/ lateral wedged footwear on coronal plane knee moments during running, showed that laterally wedged running footwear were associated with significant reductions in the peak KAM. Nigg et al., (2003) examined the effects of medial, lateral and neutral shoe inserts on knee joint moments during heel-toe running. Compared with the neutral insert condition, the maximal 
93 However, whilst the effects of foot orthoses on the biomechanics the knee joint during gait

94

95 have been examined previously, there has yet to be any investigation which has collectively explored the effects of medial and lateral orthoses on the kinetics of the patellofemoral and tibiofemoral joints during running. Therefore, the aim of the current investigation was to examine the effects of orthoses with a $5^{\circ}$ medial and lateral wedge on knee joint kinetics during the stance phase of running. A clinical investigation of this nature may provide insight into the potential efficacy of wedged foot orthoses for the prevention of knee pathologies in runners. The current investigation tests the hypotheses that medial orthoses will reduce patellofemoral joint loading and lateral orthoses will reduce the magnitude of the KAM during the stance phase of running.

\section{Methods}

\section{Participants}

Twelve male runners (age $26.23 \pm 5.76$ years, height $1.79 \pm 0.11 \mathrm{~cm}$ and body mass $73.22 \pm$ $6.87 \mathrm{~kg}$ ) volunteered to take part in this study. The sample was based on previous analyses, which have examined the effects of wedged orthoses on lower extremity kinetics during running (Almonroeder et al., 2015; Lewinson et al., 2013). All participants identified as recreational runners, who trained a minimum of 3 times/week completing a minimum of 35 $\mathrm{km} /$ week. All participants were also free from knee pathology at the time of data collection and had not previously had any knee surgery. The participants provided written informed consent in accordance with the principles outlined in the Declaration of Helsinki. The procedure utilized for this investigation was approved by the University of Central Lancashire, Science, Technology, Engineering and Mathematics, ethical committee (REF $357)$. 
Orthoses

119 Commercially available full-length orthoses (Slimflex Simple, High Density, Full Length, 120 Algeos UK) were examined in the current investigation (Figure 1-2). The orthoses were made 121 from ethylene-vinyl acetate with a shore A rating of 65 and had a heel thickness of $11 \mathrm{~mm}$ 122 including the additional wedge. The orthoses were able to be modified to either a $5^{\circ}$ varus or valgus configuration which in two separate components spanned the full length of the device (Figure 1-2). To ensure consistency each participant wore the same footwear (Asics, Patriot 6). The experimental footwear had a mean mass of $0.265 \mathrm{~kg}$, heel thickness of $22 \mathrm{~mm}$ and heel drop of $10 \mathrm{~mm}$. The order that participants ran in each orthotic condition was counterbalanced.

Procedure

Participants ran at $4.0 \mathrm{~m} / \mathrm{s}( \pm 5 \%)$, striking an embedded piezoelectric force platform (Kistler, 134 Kistler Instruments Ltd., Alton, Hampshire) with their right (dominant) foot (Sinclair et al., 135 2014a). Running velocity was monitored using infrared timing gates (Newtest, Oy 136 Koulukatu, Finland). The stance phase was delineated as the duration over which $20 \mathrm{~N}$ or greater of vertical force was applied to the force platform (Sinclair et al., 2011). Runners completed five successful trials in each orthotic condition (medial, lateral and no-orthotic). 
139 Kinematic data was captured at $250 \mathrm{~Hz}$ via an eight camera motion analysis system (Qualisys

140 Medical AB, Goteburg, Sweden). Kinematics and ground reaction forces data were 141 synchronously collected. Dynamic calibration of the motion capture system was performed 142 before each data collection session.

144 Lower extremity segments were modelled in 6 degrees of freedom using the calibrated anatomical systems technique (Cappozzo et al., 1995). To define the segment co-ordinate axes of the shank and thigh, retroreflective markers were placed bilaterally onto the medial 147 and lateral malleoli, medial and lateral epicondyles of the femur. To define the pelvis 148 segment further markers were posited onto the anterior (ASIS) and posterior (PSIS) superior 149 iliac spines. Carbon fiber tracking clusters were positioned onto the shank and thigh segments. The pelvis was tracked using the ASIS and PSIS markers. The centre of the knee joint was delineated as the mid-point between the femoral epicondyle markers (Sinclair et al., 2015a), whereas the hip joint centre was obtained using the positions of the ASIS markers (Sinclair et al., 2014b). Static calibration trials were obtained allowing for the anatomical markers to be referenced in relation to the tracking markers/ clusters. The $\mathrm{Z}$ (transverse) axis was oriented vertically from the distal segment end to the proximal segment end. The $\mathrm{Y}$ (coronal) axis was oriented in the segment from posterior to anterior. Finally, the $\mathrm{X}$ (sagittal)

157 axis orientation was determined using the right hand rule and was oriented from medial to 158 lateral. 
Dynamic trials were digitized using Qualisys Track Manager in order to identify anatomical

162

163

164

165 and tracking markers then exported as C3D files to Visual 3D (C-Motion, Germantown, MD, USA). Ground reaction force and kinematic data were smoothed using cut-off frequencies of 50 and $12 \mathrm{~Hz}$ respectively with a low-pass Butterworth $4^{\text {th }}$ order zero-lag filter. Net joint moments were calculated using Newton-Euler inverse dynamics.

A previously utilized mathematical model was used to estimate patellofemoral contact force and patellofemoral contact stress during the stance phase of running (Ward \& Powers, 2004). This model has been utilized previously to successfully resolve differences in contact force and stress when wearing different footwear (Bonacci et al., 2013; Sinclair, 2014, Sinclair et al., 2016) and between orthoses (Sinclair et al., 2015b) during running. Patellofemoral contact force was estimated as a function of the knee flexion angle and knee flexion moment according to the biomechanical model described by Ho et al., (2012). Firstly, an effective moment arm of the quadriceps muscle was calculated as a function of the knee flexion angle using a non-linear equation, which is based on cadaveric data presented by van Eijden et al., (1986):

\section{Quadriceps moment arm $=0.00008 *$ knee flexion angle ${ }^{3}-0.013 *$ knee flexion angle $^{2}$ $+0.28 *$ knee flexion angle $+\mathbf{0 . 0 4 6}$}

Quadriceps force was then estimated using the below formula: 
Patellofemoral contact force was estimated using the quadriceps force and a constant:

186

Patellofemoral contact force $=$ quadriceps force $*$ constant

The constant was described in relation to the knee flexion angle using a curve fitting technique based on the non-linear equation described by Eijden et al., (1986):

191

constant $=\left(0.462+0.00147 *\right.$ knee flexion angle ${ }^{2}-0.0000384 *$ knee flexion angle $\left.^{2}\right) /(1$ $-0.0162 *$ knee flexion angle $+0.000155 *$ knee flexion angle ${ }^{2}-0.000000698 *$ knee flexion angle ${ }^{3}$ )

Contact stress (MPa) was estimated as a function of the contact force divided by the 197 patellofemoral contact area. The contact area was described in accordance with the Ho et al., (2012) recommendations by fitting a $2^{\text {nd }}$ order polynomial curve to the data of Powers et al., (1998), which documented patellofemoral contact areas at varying levels of knee flexion. 
203 Knee loading was examined through extraction of the peak KAM, peak patellofemoral 204 contact force and peak patellofemoral contact stress. Patellofemoral contact force parameters 205 were normalized $(\mathrm{N} / \mathrm{kg})$ by dividing the net values by body mass. KAM load rate $(\mathrm{Nm} / \mathrm{kg} / \mathrm{s})$ 206 was also calculated by dividing the peak KAM by the time taken. Finally, we calculated the 207 total patellofemoral contact force impulse $(\mathrm{N} / \mathrm{kg} \cdot \mathrm{s})$ using a trapezoidal function.

Means and standard deviations were calculated for each outcome measure for all orthotic conditions. Differences in knee kinetic parameters between orthotic conditions were examined using one-way repeated measures ANOVAs, with significance accepted at the $\mathrm{P} \leq 0.05$ level. Post-hoc pairwise comparisons with a Bonferroni adjustment to control type I error, were conducted on all significant main effects. Effect sizes were conducted for each main effect and for all significant pairwise comparisons, using partial eta ${ }^{2}\left(\mathrm{p}^{2}\right)$. Effect sizes were contextualized using the following guidelines; small $=0.01$, medium $=0.06$ and large $=$ 0.14 (Cohen, 1988). The data was screened for normality using a Shapiro-Wilk, which confirmed that the normality assumption was met. All statistical analyses were conducted using SPSS v23.0 (SPSS Inc., Chicago, USA).

\section{Results}




\section{Patellofemoral kinetics}

229 A significant main effect was noted for peak patellofemoral contact force $\left(\mathrm{P}<0.05, \mathrm{p} \eta^{2}=\right.$ 230 0.29). Post-hoc pairwise comparisons showed that peak patellofemoral contact force was 231 significantly greater in the lateral $\left(\mathrm{P}=0.041, \mathrm{p}^{2}=0.31\right)$ and medial $\left(\mathrm{P}=0.045, \mathrm{p} \eta^{2}=0.31\right)$ 232 configurations, in relation to the no-orthoses condition (Figure 3a; Table 1). However, there 233 was no main effect for peak patellofemoral stress $\left(\mathrm{P}<0.05, \mathrm{p}^{2}=0.17\right.$, Figure $3 \mathrm{~b}$; Table 1$)$.

235 Finally, a significant main effect $\left(\mathrm{P}<0.05, \mathrm{p \eta}^{2}=0.37\right)$ was noted for patellofemoral impulse.

236 Post-hoc pairwise comparisons showed that patellofemoral impulse was significantly greater 237 in the lateral $\left(\mathrm{P}=0.012, \mathrm{p}^{2}=0.45\right)$ and medial $\left(\mathrm{P}=0.027, \mathrm{p}^{2}=0.37\right)$ configurations, in relation 238 to the no-orthoses condition (Table 1).

$240 \quad$ Knee adduction moment parameters

241 A significant main effect $\left(\mathrm{P}<0.05, \mathrm{p \eta}^{2}=0.31\right)$ was observed for the magnitude of peak KAM.

242 Post-hoc pairwise comparisons showed that peak KAM was significantly larger in the medial 243 orthoses in relation to the lateral orthoses $\left(\mathrm{P}=0.03, \mathrm{p \eta}^{2}=0.35\right)$ (Figure $3 \mathrm{c}$; Table 1). There was 244 however, no main effect for the KAM load rate $\left(\mathrm{P}<0.05, \mathrm{p \eta}^{2}=0.12\right.$, Table 1). 
247 The aim of the present study was to examine the influence of orthoses with $5^{\circ}$ medial and

248 lateral wedges on knee joint kinetics during the stance phase of running. To the authors knowledge this represents the first investigation to collectively explore the effects of medial and lateral orthoses on the kinetics of the patellofemoral and tibiofemoral joints during running. The findings from this investigation provide partial support for the hypotheses in that lateral orthoses significantly reduced the magnitude of the peak KAM, but both medial and lateral orthoses significantly increased patellofemoral joint loading during the stance

phase of running.

Patellofemoral pain is widely acknowledged as the most common overuse running pathology

257 (Taunton et al., 2002). The current investigation showed that patellofemoral loading parameters were significantly greater when running in the medial and lateral orthotic modalities compared to running without any orthotic intervention. This observation supports the findings of Almonroeder et al., (2015) who observed increases in patellofemoral loading when running in medial orthoses, although increases patellofemoral joint kinetics when with lateral orthoses the has not been shown previously. It is important however that the statistical observations at the patellofemoral joint be contextualized in relation to the mean difference between conditions. The mean differences between conditions were relatively small, thus it is unknown whether the statistical observations are also clinically significant. Nonetheless, this finding may still be important regarding the initiation and progression of patellofemoral pain, as the patellofemoral pain symptoms are mediated through excessive patellofemoral joint loading (Farrokhi et al., 2011). Therefore, current study indicates that running with wedged 
foot orthoses as a prophylactic modality for patellofemoral pain may not be justified, although further longitudinal analyses are required before this can be clinically substantiated.

It is proposed that the mechanism responsible for the increases in patellofemoral loading in the wedged orthotic conditions was an enhanced knee flexion moment. Similar to the proposition offered by Almonroeder et al., (2015) the additional heel elevation $(11 \mathrm{~mm})$ provided by the orthotic conditions may have influenced the vector orientation of the ground reaction force so that the magnitude of the knee flexion moment, a key input parameter into the patellofemoral model was increased. Previous trials have shown that foot orthoses served to improve patellofemoral pain symptoms (Collins et al., 2008; Barton et al., 2011); the findings from the current study indicate that the clinical improvements in pain symptoms may not have been mediated through alterations in sagittal plane knee mechanics.

In addition, the current investigation also showed the peak KAM was significantly reduced in the lateral orthotic condition in relation to the medial and no-orthotic conditions. This agrees with the observations of Lewinson et al., (2013) who showed that laterally wedged running footwear significantly reduced the peak KAM. It is proposed that this observation is mediated by the effects of the lateral orthoses themselves by attenuating the magnitude of the ground reaction force moment arm about the knee joint centre. The peak KAM is considered an effective pseudo measure of compressive medial compartment loading (Birmingham et al., 2007), and is believed to be an important biomechanical predictor of the initiation and progression of radiographic knee OA (Miyazaki et al., 2002). Again, it is important to contextualize the mean differences in peak KAM between the medial and lateral orthoses which was relatively small. As such it is not known whether the statistical changes in the 
293 KAM are clinically significant. It appears that lateral orthoses may be able to attenuate the 294 risk from the kinetic parameters linked to the aetiology of medial compartment knee OA in

A potential limitation of the current investigation is that it examines healthy male runners who habitually did not wear orthotics. Firstly, as female runners are known to be more susceptible to overuse knee injuries (Ivković et al., 2007), and secondly as it is not possible to determine if the findings are generalizable to runners with existing patellofemoral pain or medial compartment knee OA. Future, analyses will help to determine the clinical efficacy of wedged orthoses as treatment modalities for runners of both sexes, with existing chronic knee injuries. A further potential drawback is the method by which patellofemoral stress was quantified. Sagittal knee mechanics as input parameters into the mathematical model do not account for the effects of coronal/ transverse plane knee kinematics on the patellofemoral joint contact area. Further advancements in musculoskeletal research are required to provide a three-dimensional model of the patellofemoral joint contact area allowing joint stress to be calculated more accurately.

In conclusion, despite the fact that the biomechanical effects of foot orthoses have been examined previously, current knowledge with regards to the effects of medial and lateral orthoses on the loads experienced by the patellofemoral and tibiofemoral joints during running is limited. This study therefore adds to the current literature in the field of clinical 
biomechanics by giving a comprehensive comparative examination of patellofemoral and tibiofemoral loading parameters during the stance phase of running in medial and lateral orthoses. The current investigation importantly showed that lateral orthoses attenuated the magnitude of the KAM but that wearing wedged orthoses increased patellofemoral loading parameters. The results from this study indicate that lateral orthoses may be effective in attenuating runners risk from medial tibiofemoral compartment $\mathrm{OA}$, but that wedges orthoses may enhance their risk from patellofemoral pain.

\section{Funding statement}

No funding.

\section{References}

1. Almonroeder, T.G., Benson, L.C., and O'Connor, K.M. Changes in patellofemoral joint stress during running with the application of a prefabricated foot orthotic. International Journal of Sports Physical Therapy. 2015; 10: 967-975.

2. Aminian, G., and Roodsary, B.R. Knee Osteoarthritis and Orthotics Treatments. Physical Medicine \& Rehabil International. 2014; 1: 2-3.

3. Barton, C.J., Menz, H.B., Levinger, P., Webster, K.E., and Crossley, K.M. Greater peak rearfoot eversion predicts foot orthoses efficacy in individuals with patellofemoral pain syndrome. British Journal of Sports Medicine. 2011; 45: 697701.

4. Birmingham, T.B., Hunt, M.A., Jones, I.C., Jenkyn, T.R., and Giffin, J.R. (2007). Test-retest reliability of the peak knee adduction moment during walking in 
patients with medial compartment knee osteoarthritis. Arthritis Care \& Research. 2007; 57: 1012-1017.

5. Boldt, A.R., Willson, J.D., Barrios, J.A., and Kernozek, T.W. Effects of medially wedged foot orthoses on knee and hip joint running mechanics in females with and without patellofemoral pain syndrome. Journal of Applied Biomechanics. 2013; 29: 68-77.

6. Bonacci, J., Vicenzino, B., Spratford, W., and Collins, P. Take your shoes off to reduce patellofemoral joint stress during running. British Journal of Sports Medicine. 2013; http://dx. doi.org/10.1136/bjsports-2013-092160, Epub ahead of print

7. Cappozzo, A., Catani, F., Leardini, A., Benedeti, M.G., and Della C.U. Position and orientation in space of bones during movement: Anatomical frame definition and determination. Clinical Biomechanics. 1995; 10: 171-178.

8. Cohen, J. (1988). Statistical Power Analysis for the Behavioral Sciences. New York, NY: Routledge Academic.

9. Collins, N., Crossley, K., Beller, E., Darnell, R., McPoil, T., and Vicenzino, B. Foot orthoses and physiotherapy in the treatment of patellofemoral pain syndrome: randomised clinical trial. BMJ. 2008; 337: 1-8.

10. Collins, N.J., Bierma-Zeinstra, S.M., Crossley, K.M., van Linschoten, R.L., Vicenzino, B., and van Middelkoop, M. Prognostic factors for patellofemoral pain: a multicentre observational analysis. British Journal of Sports Medicine. 2012; 47: 227-233.

11. Crossley, K.M. Is patellofemoral osteoarthritis a common sequela of patellofemoral pain? British Journal of Sports Medicine. 2014; 48: 409-410. 
12. Farrokhi, S., Keyak, J.H., Powers, C.M. Individuals with patellofemoral pain exhibit greater patellofemoral joint stress: a finite element analysis study. Osteoarthritis \& Cartilage. 2011; 19: 287-294.

13. Heiderscheit, B., Hamill, J., and Tiberio, D. A biomechanical perspective: do foot orthoses work?. British Journal of Sports Medicine. 2001; 35: 4-5.

14. Ho, K.Y., Blanchette, M.G., and Powers, C.M. The influence of heel height on patellofemoral joint kinetics during walking. Gait \& Posture. 2012; 36: 271-275.

15. Hopkins, W.G. New view of statistics: Effect magnitudes. Retrieved $12^{\text {th }}$ December, 2017 from http://www.sportsci.org/resource/stats/effectmag.html

16. Hsu, W.C., Jhong, Y.C., Chen, H.L., Lin, Y.J., Chen, L.F., and Hsieh, L.F. Immediate and long-term efficacy of laterally-wedged insoles on persons with bilateral medial knee osteoarthritis during walking. Biomedical Engineering Online. 2015; 14: 2-16.

17. Ivković, A., Franić, M., Bojanić, I., and Pećina, M. Overuse injuries in female athletes. Croatian Medical Journal. 2007; 48: 767- 778.

18. Kutzner, I., Trepczynski, A., Heller, M.O., and Bergmann, G. Knee adduction moment and medial contact force-facts about their correlation during gait. PLoS One. 2013; 8: e81036.

19. Lee, D.C., Pate, R.R., Lavie, C.J., Sui, X., Church, T.S., and Blair S.N. Leisuretime running reduces all-cause and cardiovascular mortality risk. Journal of the American College of Cardiology. 2014; 64: 472-481.

20. Lewinson, R.T., Fukuchi, C.A., Worobets, J.T., and Stefanyshyn, D.J. The effects of wedged footwear on lower limb frontal plane biomechanics during running. Clinical Journal of Sport Medicine. 2013; 23: 208-215. 
21. Miyazaki, T., Wada, M., Kawahara, H., Sato, M., Baba, H., and Shimada, S. Dynamic load at baseline can predict radiographic disease progression in medial compartment knee osteoarthritis. Annals of Rheumatic Disorders. 2002; 61: 617622.

22. Morgenroth, D.C., Medverd, J.R., Seyedali, M., and Czerniecki, J.M. The relationship between knee joint loading rate during walking and degenerative changes on magnetic resonance imaging. Clinical Biomechanics. 2014; 29: 664670.

23. Nigg, B.M., Stergiou, P., Cole, G., Stefanyshyn, D., Mündermann, A., and Humble, N. Effect of shoe inserts on kinematics, center of pressure, and leg joint moments during running. Medicine \& Science in Sports \& Exercise. 2003; 35: 314-319.

24. Powers, C.M., Lilley, J.C., and Lee, T.Q. The effects of axial and multiplane loading of the extensor mechanism on the patellofemoral joint. Clinical Biomechanics. 1998; 13: 616-624.

25. Sinclair, J., Edmundson, C.J., Brooks, D., and Hobbs, SJ. Evaluation of kinematic methods of identifying gait Events during running. International Journal of Sport Science \& Engineering. 2011; 5: 188-192.

26. Sinclair, J. Effects of barefoot and barefoot inspired footwear on knee and ankle loading during running. Clinical Biomechanics. 2014; 29: 395-399.

27. Sinclair, J., Hobbs, S.J., Taylor, P.J., Currigan, G., and Greenhalgh, A. The influence of different force measuring transducers on lower extremity kinematics. Journal of Applied Biomechanics. 2014a; 30: 166-172. 
28. Sinclair, J., Taylor, P. J., Currigan, G., and Hobbs, S.J. The test-retest reliability of three different hip joint centre location techniques. Movement \& Sport Sciences. 2014b; 83: 31-39.

29. Sinclair, J., Hebron, J., and Taylor, P.J. The Test-retest Reliability of Knee Joint Center Location Techniques. Journal of Applied Biomechanics. 2015a; 31: 117121.

30. Sinclair, J., Vincent, H., Selfe, J., Atkins, S., Taylor, P.J., and Richards, J. Effects of foot orthoses on patellofemoral load in recreational runners. Foot and Ankle Online Journal. 2015b; 8: 5-9.

31. Sinclair, J., Richards, J., Selfe, J., Fau-Goodwin, J., and Shore, H. The influence of minimalist and maximalist footwear on patellofemoral kinetics during running. Journal of Applied Biomechanics. 2016; 32: 359-364.

32. Trepczynski, A., Kutzner, I., Kornaropoulos, E., Taylor, W.R., Duda, G.N., Bergmann, G., and Heller, M.O. (2012). Patellofemoral joint contact forces during activities with high knee flexion. Journal of Orthopaedic Research. 2012; 30: 408415.

33. Taunton, J.E., Ryan, M.B., Clement, D.B., McKenzie, D.C., Lloyd-Smith, D.R., and Zumbo, B.D. A retrospective case-control analysis of 2002 running injuries. British Journal of Sports Medicine. 2002; 36: 95-101.

34. Tiberio, D. The effect of excessive subtalar joint pronation on patellofemoral mechanics: a theoretical model. Journal of Orthopaedic \& Sports Physical Therapy. 1987; 9: 160-165.

35. van Eijden, T.M., Kouwenhoven, E., Verburg, J., and Weijs, W.A. A mathematical model of the patellofemoral joint. Journal of Biomechanics. 1986; 19: 219-229. 
36. van Gent, B.R., Siem, D.D., van Middelkoop, M., van Os, T.A., Bierma-Zeinstra, S.S., Koes, B.B. Incidence and determinants of lower extremity running injuries in long distance runners: a systematic review. British Journal of Sports Medicine. 2007; 41: 469-480.

37. Ward, S.R., Powers, C. M. The influence of patella alta on patellofemoral joint stress during normal and fast walking. Clinical Biomechanics. 2004; 19: 10401047.

38. Waryasz, G.R., and McDermott, A.Y. Patellofemoral pain syndrome (PFPS): a systematic review of anatomy and potential risk factors. Dynamic Medicine. 2008; 7: 1-14.

39. Wise, B.L., Niu, J., Yang, M., Lane, N.E., Harvey, W., Felson, D. T., and Lewis, C.E. Patterns of compartment involvement in tibiofemoral osteoarthritis in men and women and in whites and African Americans. Arthritis Care \& Research. 2012; 64: 847-852.

40. Yamaguchi, S., Kitamura, M., Ushikubo, T., Murata, A., Akagi, R., and Sasho, T. Effect of laterally wedged insoles on the external knee adduction moment across different reference frames. PloS one. 2015; 10: e0138554.

41. Zhao, D., Banks, S.A., Mitchell, K.H., D'Lima, D.D., Colwell, C.W., and Fregly, B.J. Correlation between the knee adduction torque and medial contact force for a variety of gait patterns. Journal of Orthopaedic Research. 2007; 25: 789-797. 
Table 1: Knee kinetics (Means, standard deviations and 95\% confidence intervals) as a function of the different orthotic conditions.

\begin{tabular}{|c|c|c|c|c|c|c|c|}
\hline & \multicolumn{2}{|c|}{ No-orthotic } & \multicolumn{2}{|c|}{ Medial } & \multicolumn{2}{|c|}{ Lateral } & \\
\hline & Mean & $S D$ & Mean & $S D$ & Mean & $S D$ & \\
\hline Peak patellofemoral contact force $(\mathrm{N} / \mathrm{kg})$ & $29.61 A B$ & 9.35 & 31.81 & 9.65 & 31.29 & 9.04 & * \\
\hline Patellofemoral impulse (N/kg·s) & $2.44 A B$ & 1.1 & 2.82 & 1.37 & 2.7 & 1.25 & * \\
\hline Peak patellofemoral stress (MPa) & 8.81 & 2.68 & 9.33 & 2.71 & 9.37 & 2.54 & \\
\hline Peak KAM (Nm/kg) & 0.93 & 0.41 & 1.1 & 0.4 & $0.87 \mathrm{~A}$ & 0.34 & * \\
\hline KAM load rate $(\mathrm{Nm} / \mathrm{kg} / \mathrm{s})$ & 25.2 & 17.89 & 24.03 & 16.55 & 24.72 & 16.57 & \\
\hline
\end{tabular}

457 Key: ${ }^{*}=$ significant main effect

$458 \boldsymbol{A}=$ significantly different from Medial orthosis

$459 \quad B=$ significantly different from Lateral orthosis 


\section{$460 \quad$ List of figures}

461 Figure 1: Figure 1: Rear image of the experimental orthoses (a. $=5^{\circ}$ medial configuration and 462 b. $=5^{\circ}$ lateral configuration).

463 Figure 2: Medial and lateral images of the experimental orthoses (a. $=$ lateral view of $5^{\circ}$ 464 lateral configuration, $\mathrm{b} .=$ lateral view of $5^{\circ}$ medial configuration, c. $=$ medial view of $5^{\circ}$ 465 lateral configuration, $\mathrm{d} .=$ medial view of $5^{\circ}$ medial configuration).

466 Figure 3: Knee joint kinetics as a function of different orthotic conditions (a. = patellofemoral 467 force, b. = patellofemoral stress, c. = knee adduction moment) (Black = medial, dot = lateral, 468 grey $=$ no-orthotic) 